
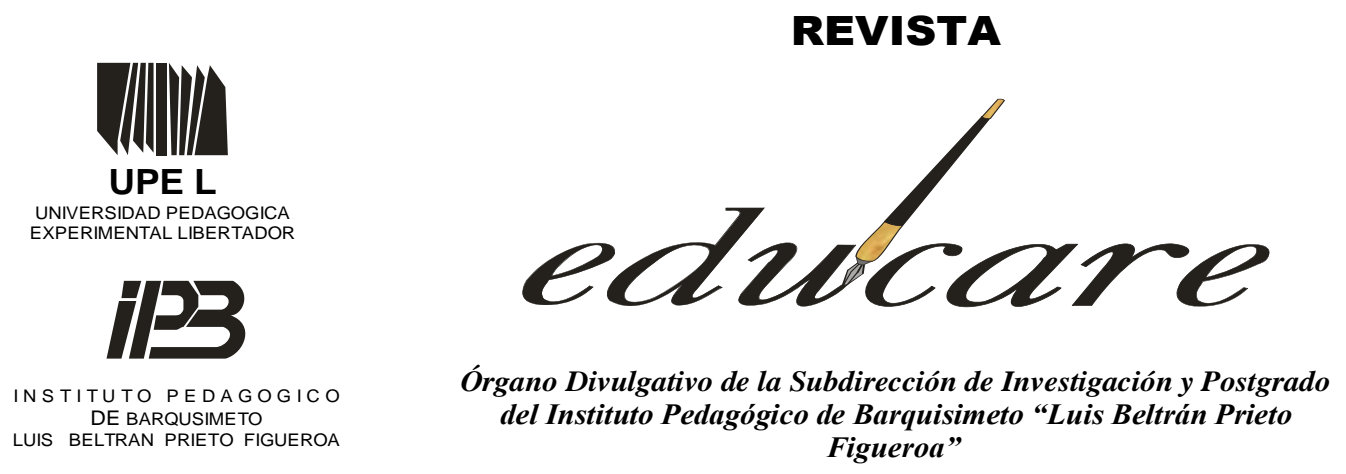

BARQUISIMETO - EDO. LARA - VENEZUELA

NUEVA ETAPA

FORMATO ELECTRÒNICO

DEPOSITO LEGAL:

PPI2O 1002LA3674

VOLUMEN 19 № 2

ISSN: 2244-7296

MAYO - AGOSTO 2015

DOCENCIA-INVESTIGACIÓN UN COMPROMISO

COTIDIANO EN LA FORMACIÓN DEL PROFESORADO UNIVERSITARIO

TEACHINGINVESTIGATION A DAILY COMMITMENT ON COLLEGE TEACHER TRAINING

MARIANGEL HERRERA *

*UNIVERSIDAD PEDAGÓGICA EXPERIMENTAL LIBERTADOR INSTITUTO PEGADÓGICO DE BARQUISIMETO (UPEL - IPB) 


\section{DOCENCIA-INVESTIGACIÓN UN COMPROMISO COTIDIANO EN LA FORMACIÓN DEL PROFESORADO UNIVERSITARIO}

\section{TEACHING-INVESTIGATION A DAILY COMMITMENT ON COLLEGE TEACHER TRAINING}

ENSAYO

Recibido:18/03/2015

\section{RESUMEN}

El mundo académico del docente se sitúa en un marco de relaciones sociales dentro del contexto que involucra $y$ articula la docencia $e$ investigación. El presente ensayo es el resultado de una revisión exhaustiva que tuvo como finalidad interpretar la formación del accionar cotidiano del docente universitario y su vinculación con la docencia y la investigación. Se utilizó la hermenéutica como método para comprender desde una perspectiva subjetiva los aportes de los autores en referencia. Se plantean reflexiones en torno al docente y su desempeño investigativo que tienden a diversificarse a la luz de las nuevas realidades sociales en un mundo lleno de complejidades. En conclusión, resulta valido pensar que la docencia y la investigación aparecen en todas las definiciones que se dan sobre las funciones básicas de la institución universitaria, la investigación para la creación del conocimiento y la docencia para su diseminación y fortaleciendo de la praxis pedagógica.

Descriptores: investigación, docencia, educación universitaria
Mariangel Herrera*

UPEL-IPB

Aceptado: 26/05/2015

\begin{abstract}
Teacher's academis world is located within a framework of social relations within the context that involves and articulates teaching and research. This essay is the result of a comprehensive review that aimed to interpret the formation of the daily actions of university teachers and its relationship with teaching and research. Hermeneutics as a method to understand from a subjective perspective the contributions of the authors in question was used. Reflections on teaching and research performance tend to diversify in light of the new social realities in a world full of complexities arise. In conclusion, it is worth thinking that teaching and research are published in all definitions given on the basic functions of the university, research for knowledge creation and teaching for its dissemination and strengthening pedagogical praxis.
\end{abstract}

Keywords: Investigation, teaching, university education

\footnotetext{
* Magister en Enseñanza de la Biología (UPEL-IPB), Profesora Tiempo Completo Pregrado y Postgrado (UPEL-IPB). Representante Departamental ante el Consejo Técnico Asesor de Investigación (UPEL-IPB). Coordinadora de Servicio Comunitario Programa de Biología (UPEL-IPB). mariangelvenezuela@ gmail.com
} 
"El Conocimiento se encuentra en un constante proceso de construcción, desconstrucción y reconstrucción"

Carlos Sluzki

\section{INTRODUCCIÓN}

Un aspecto fundamental dentro de la cotidianidad profesional docente es el de reflexionar sobre el quehacer pedagógico y en la forma de hacer cada vez más efectiva la labor como maestros, rompiendo con esquemas tradicionales y proponiendo nuevas alternativas de enseñanza donde los estudiantes sean partícipes de la dinámica académica y protagonistas de su propio conocimiento.

Ante tales circunstancias, Flores (2004), plantea que es necesario aceptar que desde la educación básica hasta la superior se requieren programas emergentes para reorientar la acción docente, hacia aquella que vaya encaminada a la formación de personas calificadas y competitivas que protagonizarán los nuevos escenarios de la dinámica socioeconómica, política y científico tecnológica nacional y mundial.

Es importante resaltar que, una sociedad basada en el conocimiento, la producción de las Universidades como unidades de cooperación con autonomía que tienen la finalidad de buscar la verdad en la comunidad de investigadores y discípulos, desde el escenario de su misión de generar y transferir conocimientos, puede y debe contribuir a la configuración de dicha sociedad, con un modelo educativo coherente, orientado a fortalecer la formación de profesionales a través de la investigación.

En este sentido, el desempeño académico supone concebir las funciones de docencia e investigación como interdependientes, de suma importancia y merecedoras de estímulo por cuanto en su conjunto sintetizan la misión de la institución, a partir de una visión compartida respecto de las demandas y exigencias de la sociedad. En este contexto de interdependencia, las funciones de docencia e investigación adquieren un carácter simbiótico, no sólo son complementarios sino interdependientes.

En este sentido, Porlán (2003), sostiene que la docencia y la investigación conforman dos de las funciones básicas del profesorado universitario, que deberían convertirse en los ejes dinamizadores, en la cotidianidad del docente. Por lo tanto, son 
nuevos desafíos y retos que debe aprehender el docente-investigador universitario en su vida diaria, cambios paradigmáticos, superar obstáculos, buscar nuevos horizontes más cerca de lo humano y mediaciones simbólicas, desenvolverse en la incertidumbre más que en la certeza, convertirse en transmisor más de preguntas que dé respuestas.

El escenario descrito representa un gran reto en la formación de profesionales de la educación para la investigación, generación y aplicación desconocimiento innovador, que se constituye en una prioridad de la problemática educativa en aras de la consolidación del campo disciplinario de las ciencias de la educación y de la intervención de los complejos problemas que le afectan en sí misma y en vinculación con la realidad social que la circunscribe.

\section{DESARROLLO}

La educación universitaria es considerada como una red compleja de expectativas, tareas y comunicaciones. El hecho de ser profesor universitario hace partícipe de esta red con independencia, si la investigación sostiene la enseñanza o si compiten dentro de un mismo saco, la enseñanza y la investigación están enlazadas en una misma red, como lo están la administración, las tutorías y demás funciones universitarias.

La investigación representa un factor de vital importancia en la búsqueda del saber y de nuevos conocimientos en las diferentes ramas, tanto naturales como humanísticas, para el discernimiento cada vez más amplio y profundo de la realidad, siendo el eje de toda institución de educación universitaria en la promoción del conocimiento, pues es el instrumento por excelencia a través del cual se puede reflejar la realidad de un país, de la comunidad y de la misma institución que la promueve, igualmente es la vía para proponer o solucionar problemáticas, generar teorías, entre otras aplicaciones.

Tomando como punto focal estas afirmaciones y en la búsqueda de orientar la gestión del docente universitario, se hace necesario revisar la diversidad de problemas asociados a la docencia universitaria a fin de enfrentarlos con una visión pragmática y estratégica. En opinión de Medina (2008), la práctica de la docencia se ha visto inmersa en una severa crisis que refleja una escasa experiencia de investigación que tenga que ver con 
la realidad, relacionada con la planificación y la comunicación.

\section{Docencia- Investigación, Un escenario para la Construcción de saberes en el contexto universitario}

El desarrollo de una praxis investigativa, consustanciada con la docencia, además de suponer el desarrollo profesional del docente universitario en su especialidad, significa disponer del recurso humano para desarrollar universidades con investigadores nóveles y activos que dedican una parte significativa de su tiempo a la investigación.

Al respecto Garagozzo (2007) plantea que las universidades, “...deben realizar notables esfuerzos para convertirse en escenarios de trasferencia significativa, construcción permanente de conocimientos y del ejercicio de los más elevados valores; donde se integre lo filosófico, lo científico y lo estético" (p.29).

Para la existencia de este contexto universitario es necesario contar con: (a) un programa permanente de formación y actualización de docentes investigadores, (b) la creación, renovación, crecimiento y mantenimiento de la infraestructura de bibliotecas, laboratorios y locales para la investigación, (c) la implantación de políticas y programas de financiamiento para la investigación, (d) la creación y mantenimiento de un programa de publicaciones periódicas que permita la divulgación de los resultados de la investigación, (e) la creación de grupos multidisciplinarios y de líneas de investigación, entre otros. Esto último permitirá contar con un nivel aceptable de calidad investigativa y de buena docencia.

El carácter instrumental del proceso investigativo, es visto por Bravo (2003) como una acción creadora de nuevos conocimientos, que se identifica con la misión universitaria de promover, generar y difundir conocimientos por medio de la investigación en todos los campos que le competen y, al mismo tiempo, como parte de los servicios que la Universidad ha de prestar a la comunidad, al entregar en sus planes de formación profesional las competencias técnicas adecuadas que permitan a los egresados contribuir al desarrollo cultural, social y económico de las sociedades.

En este sentido, es importante resaltar lo planteado por Stenhouse (citado en Cerda, 2007) “... la investigación no solo se debe reducir en producir mecánicamente un conjunto de conocimientos, sino que debe constituirse en un proceso válido para construir, 
reconstruir, analizar, explorar, explicar, comprender y descubrir el conocimiento..." (P 18). Es así como la investigación en la formación docente se deberá establecer como un proceso reflexivo, crítico y transformador de la sociedad.

Por consiguiente, la investigación se convierte en una aliada en la formación docente, por cuanto posibilita la relación entre el pensamiento y las actuaciones para coadyuvar al desarrollo personal, social y profesional del profesorado universitario y de los estudiantes.

Por lo tanto, son nuevos desafíos y retos que debe aprehender el docente universitario en su vida diaria, cambios paradigmáticos, superar obstáculos, buscar nuevos horizontes más cerca de lo humano y mediaciones simbólicas, desenvolverse en la incertidumbre más que en la certeza, convertirse en transmisor más de preguntas que dé respuestas.

En relación a lo planteado, es oportuno citar a Morín (1999), quien señala que para hacer frente a los retos de la enseñanza se requiere una reingeniería del pensamiento. En consecuencia, la formación docente demanda implementar acciones que procuren la confrontación de la teoría y la práctica, para situar al ser humano en situaciones de la vida que impulsen a la búsqueda de acuerdos, cambios de actitud mutuos, responsabilidad compartida, aprovechamiento del ambiente de aprendizaje y enseñanza, y las distintas situaciones que van emergiendo el día a día.

Como consecuencia, Cabrera ( 2003), plantea que los procesos de formación de los docentes universitarios, en el marco de las grandes exigencias que impone la sociedad se debe tomar en cuenta las perspectivas epistemológicas de la enseñanza universitaria basadas en una visión positivista con restringida concepción del ser y del hacer del profesor universitario, quien generalmente reduce su acción de la enseñanza a la transmisión del saber, con carácter rutinario- libresco, es decir, una práctica de la enseñanza centrada en el contenido teórico, el profesor lo comunica y el estudiante lo recibe, en la función del profesorado debido a que constituyen un elemento necesario de reflexión para ayudar a mejorar la práctica educativa.

En función de lo anterior, la formación de los docentes mediante la investigación requiere la integración del proceso enseñanza-aprendizaje que le permita desarrollarse en la 
disciplina que enseña. Desde luego que, un docente universitario que no realice investigación en la disciplina que enseña, es inaceptable, pero también es cierto que este docente no se decreta, se forma. Ambos perfiles profesionales se construyen mediante un proceso más o menos largo de formación teórica, reflexión, abstracción y práctica concreta.

Es válido pensar que en el escenario educativo a nivel superior, la conjugación de factores económicos, políticos y sociales, juegan un papel determinante en la función docente. No basta con el qué hacer, sino con el cómo hacerlo y con qué, para abordar las exigencias de un entorno tan vituperado, probablemente, allí radica gran parte de la problemática educativa, sobre lo cual Cruz (2002), señala que la necesidad de invertir en capacitación, a través de cursos y buenos libros, es prioridad para alcanzar el éxito profesional.

En este marco de reflexión es determinante describir el escenario actual donde se desempeña el educador universitario venezolano, considerando los parámetros económicos, políticos, sociales y personales en los cuales está imbuida su actividad.

Hoy en día, mientras el país se debate entre una crisis económica que arrastra a todos los factores del entorno, el sistema educativo, por su fragilidad y connotada esencia política, no puede aislarse a la misma. Las solapadas intervenciones a las universidades públicas, la no cancelación de los compromisos laborales y la retórica populista, perfilan un escenario difícil para quienes se desempeñan como profesionales de la Educación universitaria.

El Estado venezolano tiene la responsabilidad de definir, dentro de un proyecto de alcance nacional, los lineamientos políticos y las estrategias dirigidas a potenciar la educación como un bien público de gran trascendencia social, dándole a la figura del educador el lugar que durante décadas ha reclamado y que operativamente, se ha ganado.

En este nuevo contexto, Rugarcía (2004) plantea que se hace necesaria una reflexión acerca del quehacer y de los nuevos perfiles docentes requeridos, dada la inclusión de actividades de investigación como parte fundamental de la formación docente, se exigirán altos estándares de calidad y competitividad profesional y personal, materializado en conocimiento profundo, habilidades, adecuado perfil de personalidad, destrezas para manejar nuevas tecnologías, proactivos y diligentes, entre otros aspectos. 
En concordancia con lo planteado por el autor antes citado, el ejercicio de la praxis investigativa por parte del docente universitario supone como requisito fundamental, la formación, especialización y actualización del mismo en un área del conocimiento determinada. Por tanto, el docente universitario debe saber qué aprender, cómo aprender y qué investigar. En el proceso de formación de este docente se encuentran dos procesos paralelos: por un lado, el referido al conjunto de experiencias y conocimientos en un campo específico, lo cual le permite cumplir a cabalidad con su rol; por el otro, la adquisición y desarrollo de habilidades y destrezas investigativas para realizar con éxito su labor de investigación.

Para asumir estos procesos, el docente debe incorporarse a un proceso de desarrollo profesional relacionados con cursos de especialización, maestría y doctorado, lo cual conducirá no sólo a convertirlo en un especialista en el área de conocimiento respectivo, sino que lo proveerá del dominio conceptual y procedimental adecuado para hacer investigación.

Es importante resaltar lo establecido por la UNESCO (Organización de las Naciones Unidad para la Educación, la Ciencia y la Cultura) en la Conferencia Mundial Sobre Educación Superior: La nueva dinámica de la educación superior y la investigación para el cambio social y el desarrollo. (2009), en donde se aprobaron grandes propuestas para el progreso de la educación universitaria en la cual el profesorado tiene la gran responsabilidad de cumplir funciones en el área didáctica e investigativa. En ningún otro momento de la historia ha sido más importante que ahora la inversión en los estudios superiores, por su condición de fuerza primordial para la construcción de sociedades del conocimiento integradoras y diversas, para fomentar la investigación, la innovación y la creatividad.

Interpretando lo planteado por la UNESCO (ob.cit), en esta nueva concepción de la calidad de la docencia universitaria, las dimensiones que se trazan son: eficiencia, eficacia, disponibilidad de los recursos, procesos, y relevancia, sin embargo, no es fácil producir cambios porque el cambio implica modificar actitudes, creencias, conceptos y comportamientos. Para que el cambio se logre con éxito, las acciones de los docentes universitarios deben conjugar adecuadamente sus funciones de docencia investigación y 
extensión desde una perspectiva que privilegia la profesión como el quehacer cotidiano que exigen reflexión, creación y transformación permanente.

En consecuencia, las instituciones universitarias tienen asignados en sus objetivos, el ser centros de generación de investigación, lo cual está contemplado en su reglamentación, como lo señala la Ley Orgánica de Educación (1980), en su Art. 27:

Fomentar la investigación de nuevos conocimientos e impulsar el progreso de la ciencia, la tecnología, las letras, las artes y demás manifestaciones creadoras del espíritu en beneficio del bienestar del ser humano, de la sociedad y del desarrollo independiente de la nación (p.13).

Por consiguiente, las universidades tienen el compromiso de consolidar su labor académica y científica, además de coadyuvar en la gestión del conocimiento y responder ante el país por la formación docente. Según Filloux (2004), las universidades son entidades educativas al servicio de la sociedad con el propósito de promover la formación de sujetos deliberantes que asuman una racionalidad compleja para el cuestionamiento de realidades, construir el conocimiento a partir del a intersubjetividad y la dialéctica en la tarea de buscar la verdad, afianzar su valores trascendentales, propiciar experiencias de aprendizaje significativas y una educación ajustada a la realidad de su entorno social.

\section{REFLEXIONES FINALES}

La investigación es una fuente de saberes, la docencia es tarea fundamental para el desarrollo intelectual del individuo, unos de sus compromisos es la transmisión de conocimientos y desarrollo de destrezas. El proceso de enseñanza debe ser creativo, dinámico y reflexivo, por lo tanto al vincular la investigación y la docencia surge un cúmulo de discusiones reflexivas y planteamientos en torno a las diferentes temáticas que se pueden abordar en un trabajo investigativo. La Docencia y la Investigación representan un trabajo viviente de nutridas experiencias extraídas de la realidad de una sociedad.

La docencia y la investigación han sido centro de interés tanto para los responsables en política universitaria como para investigadores. A lo largo de las últimas décadas se ha realizado un considerable esfuerzo en tratar de establecer la naturaleza de la relación entre la docencia y la investigación. Uno de los principios básicos sostenidos por gran número de 
profesores universitarios es que entre docencia e investigación existe una estrecha relación, asegurar la calidad de estas dos funciones es una de las preocupaciones de los gobiernos y de la propia institución universitaria: de los gobiernos para proponer programas de calidad y de las instituciones universitarias para implementar dichos programas y promover la innovación en su profesorado.

Para concluir todo docente debe saber que la investigación no es lujo es una necesidad, no es posible conseguir una docencia crítica, creativa sin una búsqueda incesante de conocimientos desconectada de la docencia, la investigación académica puede convertirse en un ejercicio intelectual sin impacto social inmediato. Por eso se hace necesario integrar ambos aspectos del quehacer académico en la práctica cotidiana del docente universitario no como actividades opuestas sino interdependientes y complementarias, la producción del conocimiento educativo, tiene que enfrentar los desafíos de los grandes cambios en la ciencia, la tecnología y la sociedad. Cambios que exigen reorientar los procesos de formación de investigadores.

\section{REFERENCIAS}

Bravo, G. (2003) La UMCE, la investigación universitaria yla sociedad del conocimiento. Revista Intramurus Universidad de Chile. 13 (12) [Revista en línea]. Disponible en: http://www.umce.cl/revistas/intramuros/intramuros_n12_a12.html.[Consulta: 2015, enero 27].

Cerda, H. (1999).La investigación Formativa en el Aula. La Pedagogía como Investigación. Bogotá: Cooperativa Editorial Magisterio.

Cruz, C. (2002). El éxito profesional depende en $85 \%$ de la actitud individual. El Nacional, p. A-4.

Flórez, Ochoa R. (2004).Hacia una pedagogía del conocimiento. Bogotá. Editorial: McGrawHill.

Filloux, J. (2004). Intersubjetividad y Formación. El Retorno sobre Sí Mismo. Buenos Aires: Centro de Publicaciones Educativas.

Garagozzo, A. (2007). La Complejidad de los Saberes y Haceres Docentes desde la Rutina a la Cotidianidad. Visión del Ser que Construye el Saber y el Saber Hacer. Venezuela: Subdirección de Investigación y Postgrado de la UPEL-IPB.

Ley de Universidades (1970). Gaceta Oficial No. 1429. Extraordinario de Fecha 8 de Septiembre de 1970. 
Ley Orgánica de Educación (1980). Gaceta Oficial de la República de Venezuela, 2635 (Extraordinario) Julio 26.

Medina, A. (2008). Formación de Formadores y empleo del ordenador en la enseñanza. Fondo Social Europeo, UNED. Madrid.

Morín, E. (1999). Una Nueva Civilización para el Tercer Milenio. Madrid: Tendencias Siglo XXI, N9.

Porlán, R. (2003). El maestro como investigador en el aula. Investigar para conocer, conocer para enseñar. México: Centro de estudios sobre la universidad

Rincón, C. (s.f.) La formación de investigadores en educación:retos y Perspectivas para América Latina en el siglo XXI.Revista Iberoamericana de Educación. [Documento en línea].Disponible en: http://www.rieoei.org/deloslectores/595Rincon.pdf. [Consulta: 2014, Julio 27].

Rugarcía, A. (2004). La evaluación de la función docente. Revista Educación Superior, XXIII (3) No. 91. México.

UNESCO (2009). Conferencia Mundial sobre la Educación Superior: La nueva dinámica de la educación superior y la investigación para el cambio social y el desarrollo. Sede de la UNESCO, París. Disponible en: http://www.iesalc.unesco.org.ve/index. [Consulta 2014, Diciembre 10].

UPEL (2000) Políticas de Docencia. Vicerrectorado de Docencia. Caracas.

UPEL (2003). Manual de Trabajos de Grado de Especialización y Maestría y Tesis Doctorales. Caracas. Autor.

Vásquez, C. (2008). El Docente investigador, una salida a la crisis. Ponencia presentada en las I Jornadas de Investigación Socio educativa. 